\section{Immediate intraprocedural bleeding: true 'complication' of cold snare polypectomy?}

We have read with great interest the systematic review and meta-analysis by Jegadeesan et al, comparing the outcomes of hot snare and cold snare polypectomy (CSP) for 4- to $10-\mathrm{mm}$ colorectal polyps [1]. The results suggest a higher incidence of overall complications with CSP, attributed to a higher immediate postpolypectomy bleeding rate [IPB; $6.6 \%$ vs $3.7 \%, P=0.03]$. We feel, however, that intraprocedural bleeding (IPB) is an unfair basis upon with to make safety comparisons between the two techniques. Easily treatable and virtually inconsequential capillary oozing occurs naturally following CSP due to absence of coagulation current [2]. Importantly, in none of the three included trials did IPB cause the CSP to be aborted nor did it alter procedural management (ie need for blood transfusion and/or hospital admission). Therefore, we question whether this phenomenon is significant enough clinically to be called an "adverse event" and counted in complication statistics [3].

From an efficacy perspective, a concern is that IPB may obscure the endoscopic view and could distract from meticulous inspection of the polypectomy site. This could theoretically enhance the chance of inadvertent incomplete resection, although the current literature does not appear to support this notion. CSP of small polyps is indeed a one-step, "close and cut" procedure, which focuses on snaring at least a 1- to 2-mm clear margin of normal tissue so that a negative resection margin can be assured.

Another potential issue is that treatment of IPB (eg by hemostatic clipping) could increase costs and procedure time for CSP. As yet, no uniform interventional threshold can be recommended for treating IPB and benefits associated with this practice remain uncertain. We believe that it may be more prudent to prevent rather than treat IPB, adopting one or more of the following anecdotal measures: a) use of a thin-wire dedicated cold snare, creating less damage to the submucosal vessels [4], b) injection of submucosal saline prior to CSP (ie; cold endoscopic mucosal resection, which was highlighted by the relatively low IPB rate $(3.6 \%)$ in our study, attributable to a tamponade effect exerted on submucosal vessels [5]); and c) adding epinephrine in the injectate, which could further reduce incidence of IPB due to its vasoconstrictive action.

Obviously, data are yet insufficient to address differences in terms of "true" complications (ie; delayed post-polypectomy bleeding and perforation), as none of the three trials was adequately powered in that sense. Indirect evidence, however, supports a more favorable profile for CSP in terms of delayed bleeding, including the fact that injured submucosal arteries can be demonstrated in fewer than $5 \%$ of cases when using a dedicated cold snare and CSP appears to be safe in patients on anti-coagulants [4]. Last but not the least, perforation and post-polypectomy syndrome are negligible risks without use of diathermy.

In conclusion, more prospective research is needed to clarify safety comparisons between CSP and hot polypectomy, also gauging the differential clinical impact of IPB. At this stage, metaanalyses comparing the two techniques are great to provide a sense of reassurance for practicing endoscopists, showing that both techniques are effective and safe for the removal of small polyps.

\section{Competing interests}

None
The authors

Vasilios Papastergiou' ${ }^{1}$, Gregorios A.

Paspatis $^{2}$, Konstantina D. Paraskeva ${ }^{1}$

1 Gastroenterology Department, “Konstantopoulio-Patision” General Hospital of Nea lonia, Athens, Greece

2 Gastroenterology Department, "Venizelion" General Hospital of Heraklion, Heraklion, Greece

\section{Corresponding author}

\section{Vasilios Papastergiou, MD}

Gastroenterology Department,

“Konstantopoulio-Patision" General Hospital of Nea Ionia, Agias Olgas 3 -5, 14233, Nea Ionia, Athens, Greece

Fax: 2132057048

vasi.pap@hotmail.com

\section{References}

[1] Jegadeesan R, Aziz M, Desai M et al. Hot snare vs. cold snare polypectomy for endoscopic removal of 4-10 mm colorectal polyps during colonoscopy: a systematic review and meta-analysis of randomized controlled studies. Endosc Int Open 2019; 7: E708-E716

[2] Paspatis GA, Tribonias G, Konstantinidis K et al. A prospective randomized comparison of cold vs hot snare polypectomy in the occurrence of postpolypectomy bleeding in small colonic polyps. Colorectal Dis 2011; 13: e345-348

[3] Cotton PB, Eisen GM, Aabakken L et al. A lexicon for endoscopic adverse events: report of an ASGE workshop. Gastrointest Endosc 2010; 71: 446 - 454

[4] Makino T, Horiuchi A, Kajiyama M et al. Delayed bleeding following cold snare polypectomy for small colorectal polyps in patients taking antithrombotic agents. J Clin Gastroenterol 2018; 52: 502 - 507

[5] Papastergiou V, Paraskeva KD, Fragaki M et al. Cold versus hot endoscopic mucosal resection for nonpedunculated colorectal polyps sized 6-10 mm: a randomized trial. Endoscopy 2018; 50: 403 -411 
Bibliography

DOI http://dx.doi.org/10.1055/a-0966-8572

Endoscopy International Open 2019; 07: E1031-

E1032

(c) Georg Thieme Verlag KG

Stuttgart · New York

elSSN 2196-9736

다(i) $(5)$ 